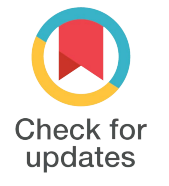

DOI: $10.29252 / \mathrm{ijcp}-26691$

\section{Effect of Retrograde Autologous Priming on Clinical Outcome of Cardiopulmonary Bypassing on Patients Undergoing Coronary Artery bypass Grafting}

\author{
Musa Rafiee ${ }^{1}$, Mohammad Zia Toutounchi ${ }^{2}$, Rezvan Yazdani \\ Soodejani ${ }^{3}$, Nooredin Mohamadi ${ }^{1, *}$ \\ ${ }^{1}$ Department of Critical Care Nursing, Center for Nursing Care Research, Nursing \\ and Midwifery Faculty, Iran University of Medical Sciences, Tehran, Iran \\ ${ }^{2}$ Heart Valve Disease Research Center, Rajaie Cardiovascular Medical \& Research \\ Center, Iran University of Medical Sciences, Tehran, Iran \\ ${ }^{3}$ Department of Nursing, School of Nursing and Midwifery, Shahrekord University \\ of Medical Sciences, Shahrekord, Iran \\ * Corresponding author: Nooredin Mohammadi, Department of Critical Care Nursing, \\ Center for Nursing Care Research, Nursing and Midwifery Faculty, Iran University of \\ Medical Sciences, Tehran, Iran. Tel: +98-9122001587, E-mail: \\ nooredin.mohammadi@yahoo.com
}

\begin{tabular}{l} 
Submitted: 10-08-2019 \\
Accepted: 05-11-2019 \\
\hline Keywords: \\
Blood Transfusion \\
Autologous \\
Cardiopulmonary Bypass \\
\hline C) 2020. International Journal \\
of Cardiovascular Practice.
\end{tabular}

\section{INTRODUCTION}

Cardiopulmonary bypass (CPB) is a standard and low mortality-associated method used across the world. This method allows cardiac surgery to be performed in a bloodless environment but preparing the $\mathrm{CPB}$ circuits before operation requires to prime it by about $1500 \mathrm{ml}$ of crystalloid solution which is a cause of significant 
hemodilution. This hemodilution leads to disrupted homeostasis and increased need for blood transfusions during and after surgery in patients [1]. Decreased blood viscosity, improved microcirculation and enhanced venous return with the following increase in cardiac output are known benefits of moderate hemodilution. However, severe hemodilution with subsequent anemia may resulting in ischemic organ injury.

Decreased hematocrit results in decreased blood oxygen content and oxygen delivery to the tissues. Hypoxia leads to increased tissue damage and mortality [2]. Particularly, anemia in heart surgery, when hematocrit reaches less than $20 \%$, is an important risk factor for stroke, renal failure, and increased mortality [3]. Besides, bleeding and anemia after the operation cause some specific issues that reduce the body's ability to provide adequate perfusion to the body's major organs, such as the brain, heart, and kidneys, leading to failure of these organs exacerbated by hemodilution. However, resolving this problem using blood components transfusion also causes issues such as blood type incompatibility $(1: 10,000)$, blood-borne infections $(1: 350,000)$, lung damage $(1: 50,000)$, cerebral edema and kidney dysfunction [4]. Reducing the volume of prime is the technique that decreases hemodilution and blood transfusion during cardiopulmonary bypass. Retrograde autologous priming (RAP), developed in 1998 by Rosenberg, is a non-pharmacological method for keeping blood. In this method, before the initiation of the bypass, blood from the arterial and venous cannulas is directed toward the bypass circuit and the blood inside the crystalloid circuit is directed toward a bag [5].

By increasing blood osmotic pressure and reducing pulmonary edema, RAP improves patient outcomes and reduces the postoperative mechanical ventilation time $[6,7]$. Hwang et al's study on regional brain oxygenation showed that RAP increased oxygenation of the brain by reducing hemodilution [8]. Severdija et al. reported that hemofiltration and bleeding during and 24 hours after the surgery were lower in the RAP group than the control, and intraoperative hematocrit was higher in the RAP group than the control and the total number of patients and the units of blood given were lower in the RAP group than the control [9]. A study in Sao Paulo, Brazil showed that RAP increases hematocrit during surgery, especially in people with anemia, followed by decreased hemolytic reactions due to decreased blood transfusion. However, some results reported the RAP as a useful method while some others not [10]. Kearsey et al. study showed that there were no differences in blood transfusion, platelet, frozen plasma, 30-day mortality, reoperation, and the amount of hemoglobin between the RAP and the control group [11]. Another study showed that this technique did not affect the clinical outcome of patients [12]. In a prospective study conducted by Nanjapa et al., RAP did not affect hemoglobin, hematocrit level and blood product transfusion [13]. A retrospective cohort study demonstrated that RAP was ineffective in bleeding control in the operating room while performing this technique caused hemodynamic instability [14].

According to the results of studies, there were no pieces of evidence over the usefulness of RAP before cardiopulmonary bypass regarding the clinical outcomes, therefore, further studies are needed to determine the effects of RAP on the clinical outcomes. We aimed to investigate the effect of RAP on the clinical outcomes of cardiopulmonary bypass in patients undergoing coronary artery bypass graft ( $\mathrm{CABG})$.

\section{METHODS}

This study is a randomized clinical trial and received the ethical approval from the ethics committee of Iran University of Medical Sciences and Rajaei Hospital (IR.IUMS.REC1395.9311584002). Written consensus has been obtained from all the patients.

\section{Study Population}

The research population in this study includes all patients undergoing $\mathrm{CABG}$ using cardiopulmonary bypass in Rajaei Hospital. Adult patients undergoing CABG without previous heart surgery, aged 18 to 70 years, weight over 45 and less than $100 \mathrm{~kg}$, hemoglobin levels over 8.5 and less than $14 \mathrm{mg} / \mathrm{dL}$, and left ventricular ejection fraction over $35 \%$ were enrolled in the study. Patients undergoing emergency surgery, having preoperative coagulation problems, undergoing mechanical ventilation prior to surgery, patients with carotid plaque leading to stenosis more than 60\%, COPD patients, patients with serum creatinine levels over $1.2 \mathrm{mg} / \mathrm{dL}$, using hemo-filter, cell saver and patients with bleeding after surgery were excluded from the study. If mean arterial pressure decreases to less than $60 \mathrm{mmHg}$ during the operating of RAP, the technique is stopped and the data were excluded from the study. Eligible patients were randomly assigned to either the retrograde autologous priming or conventional priming. The randomization was performed according to a random number sequence generated by the computer.

\section{Intraoperative Detail and CPB Management}

Anesthesia was performed in all patients using a single technique. To achieve this purpose, $10 \mu \mathrm{g} . \mathrm{kg}^{-1}$, fentanyl and $0.1 \mathrm{mg} \cdot \mathrm{kg}^{-1}$ of midazolam, and then, to maintain anesthesia, $0.5 \mathrm{mg} \cdot \mathrm{kg}^{-1} \cdot \mathrm{hr}^{-1}$ fentanyl and $1 \mu \mathrm{g} \cdot \mathrm{kg}^{-1} \cdot \mathrm{min}^{-1}$ Midazolam was administered. In patients with low EF, attempts have been made to prevent hemodynamic changes and prevent the reduction in heart rate and blood pressure and avoidance of arrhythmias. CPB was performed in all patients with a roller pump and nonpulsatile flow (2.4 L.m $\left.{ }^{2} \cdot \mathrm{min}\right)$. Mean arterial pressure was maintained above $60 \mathrm{~mm} \mathrm{Hg}$. CPB circuits were primed with $1000 \mathrm{ml}$ of ringer lactate and $500 \mathrm{ml}$ of hydroxyethyl starch. 
A median sternotomy was performed in all patients, and arterial and venous cannulation was conducted after systemic heparinization and before the onset of CPB. 300 u. $\mathrm{kg}^{-1}$ Heparin was given to maintain activated clotting time (ACT) over 480 seconds during CPB. Patients were cooled to $32{ }^{\circ} \mathrm{C}$ during aortic clamp and heated to $37^{\circ} \mathrm{C}$ after opening the aortic clamp. At the completion of the surgery, protamine was used to neutralize the effect of heparin.

\section{Study Design and Study Variable}

When the arterial cannula that had been placed in the patient's aorta was connected to the arterial line of $\mathrm{CPB}$, the patient was placed in the Trendelenburg position and clamp was removed from the arterial line so that the blood could retrogradely enter in the arterial line, oxygenator, and arterial filter and replace the fluid in them that exited from the circuit and entered the collecting bag. By coordination between perfusion and anesthetic teams, preventing the reduction of mean arterial pressure to less than $60 \mathrm{mmHg}$ and, if needed vasopressor was used to prevent hypotension.

For the implementation of RAP, a 1.4-inch line that connected the arterial route to the oxygenator (the line that returned blood to the venous reservoir) was used. This line was connected to a 1-liter tank to collect the liquid. RAP was implemented after the ACT reached over 400 seconds through three steps. In the first step recirculation line was open. This allowed blood to move reversibly from the arterial line to the arterial filter and the liquid contained in this line was transferred into the collecting bag. In the second step, the liquid in venous reservoir and oxygenator was depleted; in this step, the line between the oxygenator and arterial line was closed and the recirculation line was opened, arterial pump slowly progressed and fluid in the venous reservoir passed into the collecting bag until the volume of fluid in the venous reservoir reached approximately $200 \mathrm{ml}$. Then, the exit of the arterial filter was open and the arterial pump continued until the fluid that exited the oxygenator became sanguineous; then, the arterial pump stopped and the recirculation line was clamped. In the third step, which coincided with the start of $\mathrm{CPB}$, the fluid in the venous line entered the collecting bag until the effluent fluid became sanguineous. Afterward, the recirculation line was clamped and the fluid that was collected in the bag was maintained to be added to the system if the crystalloid fluid was needed. The tools used to collect information were two checklists one of which was used to collect demographic information including name, age, sex, body surface, height, weight, the group to which the participant was assigned, and the number of grafts. The other checklist completed during and after the $\mathrm{CPB}$ was used to collect data on total bypass duration, aortic cross-clamp and surgery duration, the duration of being connected to the mechanical ventilation device, the blood components transfused in the operating room and the intensive care unit, ejection fraction (EF) of left ventricle and changes in blood gases were recorded. Both medical and nursing staff in the ICU and the postoperative wards were blinded from the priming techniques used for this study. The data were collected during the $\mathrm{CPB}$, surgery, and hospitalization in both the intensive care unit and otherwise for both groups and recorded in the checklists.

The present study was registered in the Iranian Registry of Clinical Trials (IRCT registration number: IRCT2016073029123N1).

\section{Statistical Analysis}

All statistical analyses were performed in SPSS (Version 23.0, SPSS Inc., Chicago, IL, USA) using descriptive statistics (mean $\pm \mathrm{SD}$ ). The sample size was determined with a $95 \%$ confidence interval and $80 \%$ power, and assuming that RAP could prevent the reduction in hemoglobin by $\mathrm{d}=1$ for its effect to be considered statistically significant. It is noteworthy that in a similar study, the standard deviation of hemoglobin was estimated at $1.5(\mathrm{SD}=1.5)$ and sample size in each group was estimated 40 considering $10 \%$ dropout. Chisquare test was used to compare qualitative data (sex) and an independent t-test was used to compare quantitative data (age, height, weight, hemoglobin, haematocrit, blood components transfusion, Duration of mechanical ventilation, blood gases, Ejection Fraction). P-value $<0.05$ was considered statistically significant.

\section{RESULTS}

\section{General Characteristics}

In this study, 80 patients, assigned to two groups of 40 each: RAP and conventional priming, were studied. According to statistical analysis, there was no significant difference in age, sex, height, weight, graft number, duration of the total bypass, and duration of aortic crossclamping between the two groups $(\mathrm{P}>0.05)$ (Table 1$)$. The difference in haemoglobin $(\mathrm{mg} / \mathrm{dl})(11.45 \pm 1.17$ vs. $11.61 \pm 1.23)$ and hematocrit $(\%)(36.17 \pm 5.78$ vs. $36.39 \pm 7.03)$ before the pump was not significant between the RAP group and the conventional priming group. But, there was a significant difference in haemoglobin $(\mathrm{mg} / \mathrm{dl})(8.99 \pm 0.95$ vs. $8.65 \pm 0.97)$ and hematocrit (\%) $(28.95 \pm 3.23$ vs. $27.65 \pm 3.62)$ after the pump. Besides, during the first $30 \mathrm{~min}$ of $\mathrm{CPB}$, hemoglobin $(\mathrm{mg} / \mathrm{dl})(8.5 \pm 0.89$ vs. $7.29 \pm 0.64)$ and HCT (\%) (27.7 \pm 3.12 vs. $25.49 \pm 2.24)$ were significantly higher in the RAP group compared to the conventional priming group. This finding confirmed that hemodilution is reduced by using RAP.

\section{Comparison of the Need for Blood Components Transfusion during and After Surgery}

Transfusion of PRBC and other blood products were not significantly different during and after the operation (Table 2). 


\section{Duration of Mechanical Ventilation}

The mean duration of mechanical ventilation (h) was significantly shorter in the RAP group $(8.70 \pm 1.83)$ than the conventional priming group $(10.38 \pm 2.94)$ ( $\mathrm{P}$ $=0.003)$ (Table 3$)$.

\section{Left Ventricular Ejection Fraction}

The left ventricular EF was compared between the two groups before and after surgery. The differences in preoperative $\mathrm{EF}(\%)(47.50 \pm 7.51$ vs. $44.13 \pm 8.39)$ and postoperative $\mathrm{EF}(43.63 \pm 5.66$ vs. $42.38 \pm 6.40)$ were not statistically significant between the RAP group and the conventional priming group $(\mathrm{P}>0.05)$ (Table 4$)$.

\section{Changes in Blood Gases in Two Groups}

Changes in blood gases such as oxygen $(\mathrm{mmHg})$ and carbon dioxide $(\mathrm{mmHg})$, and acidity $(\mathrm{PH})$ in the two groups were investigated in four times, the baseline, half an hour after the onset of the pumps, at the completion of the pump, and after that. The results showed that the amounts of oxygen and carbon dioxide and changes in $\mathrm{pH}$ were not significantly different between the two groups $(\mathrm{P}>0.05)$.

Table 1. Demographic Features of Patients in Two Groups

\begin{tabular}{|c|c|c|c|}
\hline Variable & RAP & Conventional priming & Pvalue \\
\hline Age, year & $60.53 \pm 9.45$ & $62.35 \pm 10.24$ & 0.4 \\
\hline \multicolumn{4}{|l|}{ Sex } \\
\hline Female & $15(37.5)$ & $15(37.5)$ & 0.5 \\
\hline Male & $25(62.5)$ & $25(62.5)$ & 0.5 \\
\hline Height, cm & $167.5 \pm 8.79$ & $164.95 \pm 9.31$ & 0.2 \\
\hline Weight, kg & $75.38 \pm 11.8$ & $74.50 \pm 12.47$ & 0.78 \\
\hline Graft number & $3.38 \pm 0.63$ & $3.23 \pm 0.62$ & 0.2 \\
\hline Duration of aortic cross-clamping, min & $44.35 \pm 17.58$ & $44.35 \pm 17.55$ & 1.0 \\
\hline Duration of total bypass, min & $80.55 \pm 29.72$ & $83.48 \pm 26.45$ & 0.6 \\
\hline Base haemoglobin, mg/dl & $11.45 \pm 1.17$ & $11.61 \pm 1.23$ & 0.2 \\
\hline
\end{tabular}

Data in table are presented as No. (\%) or Mean \pm SD. RAP: Retrograde autologous prime

Table 2. Comparison of Blood transfusions in retrograde autologous priming and conventional Priming groups

\begin{tabular}{|ccc}
\hline & RAP & Conventional priming \\
\hline Unit of packed cell transfusion (ml) & & P value \\
\hline During cardiopulmonary bypass & $118.75 \pm 26.84$ & $275 \pm 39.83$ \\
After cardiopulmonary bypass & $106.25 \pm 26.69$ & $162.5 \pm 38.55$ \\
Unit of platelets transfusion (ml) & & 0.002 \\
\hline During cardiopulmonary bypass & $8.75 \pm 4.34$ & 0.2 \\
After cardiopulmonary bypass & $12.5 \pm 4.29$ & $13.75 \pm 5.06$ \\
Unit of FFP transfusion (ml) & & 0.4 \\
\hline During cardiopulmonary bypass & $41.25 \pm 15.18$ & $48.75 \pm 6.2$ \\
\hline After cardiopulmonary bypass & $49.5 \pm 18.9$ & $45 \pm 18.76$ \\
\hline
\end{tabular}

Data in table are presented as Mean \pm SD. RAP: Retrograde autologous prime; FFP: Fresh frozen plasma

Table 3. Comparison of Ventilator Time in retrograde autologous priming and Conventional priming Priming groups

\begin{tabular}{lccc}
\hline Variable & RAP & Conventional priming & P value \\
\hline Ventilator Time (Hour) & $8.70 \pm 1.83$ & $10.38 \pm 2.94$ & 0.003 \\
\hline Data in table are presented as Mean \pm SD. RAP: Retrograde autologous prime & &
\end{tabular}

Table 4. Comparison of Ejection Fraction in retrograde autologous priming and Conventional priming groups

\begin{tabular}{lccc}
\hline & RAP & Conventional priming \\
\hline Ejection Fraction, \% & & & \\
During cardiopulmonary bypass & $47.50 \pm 7.51$ & $44.13 \pm 8.39$ & \\
After cardiopulmonary bypass & $43.63 \pm 5.66$ & $42.38 \pm 6.40$ & 0.06 \\
\hline
\end{tabular}

Data in table are presented as Mean \pm SD. RAP: Retrograde autologous prime

\section{DISCUSSION}

Increased hemodilution during $\mathrm{CPB}$ due to decreased oxygen supply is associated with the dysfunctioning of vital organs. Hemodilution reduces the amount of coagulation-associated proteins and therefore increases bleeding during CPB. The results of this study showed a decrease in transfusions of blood components during surgery in the group that increased with RAP. The number of patients requiring transfusion of PRBC and the mean number of transfused units of PRBCs was significantly different between the RAP and the conventional priming group during the surgery.
Rosengart et al. showed that $3 \%$ of patients in the RAP group and $23 \%$ in the conventional prime group required a blood transfusion during surgery [15]. Also Shapira et al. showed that RAP caused a reduction in PRB transfusion [16]. Saczkowski's meta-analysis investigate the effectiveness of RAP in reducing transfusion of PRBCs in the adult heart surgery concluded that the number of patients receiving PRBC transfusion during operation and the entire hospital stay is reduced by this method [17]. Available findings reported a significant reduction in blood transfusions needed in patients who treated with RAP [18]. There are varying degrees of hemodilution in $\mathrm{CPB}$ that may 
offer advantages such as decreased peripheral vascular resistance, improved microcirculation perfusion, and reduced blood loss. Therefore, moderate hemodilution is highly essential to manage CPB but excessive hemodilution can affect the perfusion and damage of organs [19].

Deceased hematocrit level causes tissue hypoxia which is the most important mechanism of damage to the body organs and increased mortality [19]. The mean duration of mechanical ventilation was significantly different between the RAP group and the conventional priming group, which is consistent with the results of Foglia [7] and Trapp et al. ${ }^{6}$ study reporting RAP reduced the duration of mechanical ventilation, improved the outcome of patients, and reduced pulmonary edema through increasing blood osmotic pressure. Teman et al. showed that difference in longer than ventilation more than 48 -h was not statistically significant between intervention and control groups [19]. Trapp et al. comparatively studied RAP, conventional priming, and miniaturized $\mathrm{CPB}$. Their results showed that the duration of mechanical ventilation was not significantly different among the three groups [6].

Heart EFs were compared before and after the surgery in both groups. There was no significant difference in EF between before and after the surgery in both groups, which is consistent with Severdija [9] and Teman [20] studies and inconsistent with Teman study [21].

The changes in blood gases such as oxygen, carbon dioxide and $\mathrm{PH}$ in the two groups were investigated four times before the pump, during the pump ( 30 minutes after starting the pump), at the completion of the pump, and after the pump. The results showed that the changes in oxygen and carbon dioxide and $\mathrm{PH}$ in the two groups were statistically significantly different at none of the four intervals [22]. In analyzing lactate levels at different intervals, we could not find any significant difference between the conventional priming group and RAP group [23] which is consistent with Ancheri et al. study [24].

\section{CONCLUSIONS}

Because of the more frequent use of allogeneic blood in heart surgery than other surgeries and limitations in banked bloods, the use of lower amounts of such bloods is an essential purpose in cardiac surgery. The use of RAP can help to maintain hematocrit at higher levels, maintain hemodynamic and hemostasis status in good condition, and prevent infections associated with blood transfusions. The results of our study showed that RAP reduced hemodilution, increased hematocrit and, therefore blood transfusion during CPB. In this study, the duration of mechanical ventilation was shorter in the RAP group than the conventional priming group. According to the findings, the amount of blood transfusion, not blood products, is less during surgery. Therefore, it is recommended to consider RAP as an effective and low-cost technique of priming $\mathrm{CPB}$ circuits.

\section{Funding/Support}

This article supported by Iran University of Medical Sciences- Deputy of Research.

Conflicting Interest

The authors declare no conflict of interests.

\section{REFERENCES}

1. Cormack JE, Forest RJ, Groom RC, Morton J. Size makes a difference: use of a low-prime cardiopulmonary bypass circuit and autologous priming in small adults. Perfusion. 2000;15(2):129-35. doi: 10.1177/026765910001500207 pmid: 10789567

2. Tobian AA, Ness PM, Noveck H, Carson JL. Time course and etiology of death in patients with severe anemia. Transfusion. 2009;49(7):1395-9. doi: 10.1111/j.1537-2995.2009.02134.x pmid: 19389032

3. Nalla BP, Freedman J, Hare GM, Mazer CD. Update on blood conservation for cardiac surgery. J Cardiothorac Vasc Anesth. 2012;26(1):117-33. doi: 10.1053/j.jvca.2011.07.024 pmid: 22000983

4. Silliman CC, Paterson AJ, Dickey WO, Stroneck DF, Popovsky MA, Caldwell SA, et al. The association of biologically active lipids with the development of transfusion-related acute lung injury: a retrospective study. Transfusion. 1997;37(7):719-26. doi: $\quad 10.1046 / j .1537-2995.1997 .37797369448 . x \quad$ pmid: 9225936

5. Eising GP, Pfauder M, Niemeyer M, Tassani P, Schad H, Bauernschmitt R, et al. Retrograde autologous priming: is it useful in elective on-pump coronary artery bypass surgery? Ann Thorac Surg. 2003;75(1):23-7. doi: 10.1016/s00034975(02)04099-7 pmid: 12537187

6. Trapp C, Schiller W, Mellert F, Halbe M, Lorenzen H, Welz A, et al. Retrograde Autologous Priming as a Safe and Easy Method to Reduce Hemodilution and Transfusion Requirements during Cardiac Surgery. Thorac Cardiovasc Surg. 2015;63(7):628-34. doi: 10.1055/s-0035-1548731 pmid: 25803120

7. Foglia RP, Lazar HL, Steed DL, Follette DM, Manganaro AJ, Deland E, et al. Iatrogenic myocardial edema with crystalloid primes: effects on left ventricular compliance, performance, and perfusion. Surg Forum. 1978;29:312-5. pmid: 122613

8. Hwang J, Huh J, Kim J, Park S, Hwang J, Nahm FS, et al. The effect of retrograde autologous priming of the cardiopulmonary bypass circuit on cerebral oxygenation. J Cardiothorac Vasc Anesth. 2011;25(6):995-9. doi: 10.1053/j.jvca.2011.02.017 pmid: 21576024

9. Severdija EE, Heijmans JH, Theunissen M, Maessen JG, Roekaerts $\mathrm{PH}, \mathrm{Weerwind} \mathrm{PW}$. Retrograde autologous priming reduces transfusion requirements in coronary artery bypass surgery. Perfusion. 2011;26(4):315-21. doi: $10.1177 / 0267659111408379$ pmid: 21593085

10. Reges RV, Vicente WV, Rodrigues AJ, Basseto S, Alves Junior L, Scorzoni Filho A, et al. Retrograde autologous priming in cardiopulmonary bypass in adult patients: effects on blood transfusion and hemodilution. Rev Bras Cir Cardiovasc. 2011;26(4):609-16. doi: 10.5935/1678-9741.20110052 pmid: 22358277

11. Kearsey C, Thekkudan J, Robbins S, Ng A, Lakshmanan S, Luckraz $H$. Assessing the effectiveness of retrograde autologous priming of the cardiopulmonary bypass machine in isolated coronary artery bypass grafts. Ann R Coll Surg Engl. 2013;95(3):207-10. doi: 10.1308/003588413X135116099 56859 pmid: 23827293

12. Sun P, Ji B, Sun Y, Zhu X, Liu J, Long C, et al. Effects of retrograde autologous priming on blood transfusion and clinical outcomes in adults: a meta-analysis. Perfusion. 
2013;28(3):238-43. doi: 10.1177/0267659112474861 pmid: 23341136

13. Nanjappa A, Gill J, Sadat U, Colah S, Abu-Omar Y, Nair S. The effect of retrograde autologous priming on intraoperative blood product transfusion in coronary artery bypass grafting. Perfusion. 2013;28(6):530-5. doi: 10.1177/02676591134917 76 pmid: 23744846

14. Murphy GS, Szokol JW, Nitsun M, Alspach DA, Avram MJ, Vender JS, et al. The failure of retrograde autologous priming of the cardiopulmonary bypass circuit to reduce blood use after cardiac surgical procedures. Anesth Analg. 2004;98(5):12017, table of contents. doi: 10.1213/01.ane.0000112306.7111 3.5e pmid: 15105188

15. Rosengart TK, DeBois W, O'Hara M, Helm R, Gomez M, Lang $\mathrm{SJ}$, et al. Retrograde autologous priming for cardiopulmonary bypass: a safe and effective means of decreasing hemodilution and transfusion requirements. J Thorac Cardiovasc Surg. 1998;115(2):426-38; discussion 38-9. doi: 10.1016/S00225223(98)70287-9 pmid: 9475538

16. Shapira OM, Aldea GS, Treanor PR, Chartrand RM, DeAndrade KM, Lazar HL, et al. Reduction of allogeneic blood transfusions after open heart operations by lowering cardiopulmonary bypass prime volume. Ann Thorac Surg. 1998;65(3):724-30. doi: 10.1016/s0003-4975(97)01431-8 pmid: 9527202

17. Saczkowski R, Bernier PL, Tchervenkov CI, Arellano R. Retrograde autologous priming and allogeneic blood transfusions: a meta-analysis. Interact Cardiovasc Thorac Surg. 2009;8(3):373-6. doi: 10.1510/icvts.2008.195354 pmid: 19074909

18. Liu Y, Cui HJ, Tao L, Chen XF. [Clinical research of minimal extracorporeal circulation in perioperative blood conservation of coronary artery bypass graft]. Zhonghua Wai Ke Za Zhi. 2011;49(4):307-10. pmid: 21612694

19. Sarnowski W, Ponizynski A, Lowicki Z, Dyszkiewicz W, Paluszkiewicz L. [The effect of hemodilution and autotransfusion on diagnostic levels of digoxin in serum of patients operated on with extracorporeal circulation]. Pol Tyg Lek. 1994;49(25-26):564-5, 9. pmid: 7659610

20. Teman N, Delavari N, Romano M, Prager R, Yang B, Haft J. Effects of autologous priming on blood conservation after cardiac surgery. Perfusion. 2014;29(4):333-9. doi: 10.1177/0267659113517923 pmid: 24395680

21. Fu GW, Nie YF, Jiao ZY, Zhao WZ. Clinical applications of retrograde autologous priming in cardiopulmonary bypass in pediatric cardiac surgery. Braz J Med Biol Res. 2016;49(5):e5138. doi: 10.1590/1414-431X20165138 pmid: 27119427

22. Li M, Bertout JA, Ratcliffe SJ, Eckenhoff MF, Simon MC, Floyd TF. Acute anemia elicits cognitive dysfunction and evidence of cerebral cellular hypoxia in older rats with systemic hypertension. Anesthesiology. 2010;113(4):845-58. doi: 10.1097/ALN.0b013e3181eaaef9 pmid: 20808217

23. Society of Thoracic Surgeons Blood Conservation Guideline Task F, Ferraris VA, Ferraris SP, Saha SP, Hessel EA, 2nd, Haan CK, et al. Perioperative blood transfusion and blood conservation in cardiac surgery: the Society of Thoracic Surgeons and The Society of Cardiovascular Anesthesiologists clinical practice guideline. Ann Thorac Surg. 2007;83(5 Suppl):S27-86. doi: 10.1016/j.athoracsur.2007.02.099 pmid: 17462454

24. Ancheri SA, Rao SV. Retrograde autologous priming reduces transfusion requirements during CPB. India J Appl Res. 2016;6(8). 